\title{
As propriedades do butiá benéficas à saúde a partir da visão dos integrantes-chave da Rota dos Butiazais
}

Márcia Kaster Portelinha ${ }^{1}$, Rosa Lía Barbieri ${ }^{2}$, Teila Ceolin ${ }^{3}$

\section{Resumo}

O estudo teve o objetivo descrever o conhecimento dos integrantes da Rota dos Butiazais a respeito das propriedades do butiá que são benéficas à saúde. Pesquisa qualitativa, de abordagem descritiva, com a coleta de dados em março e abril de 2020. Foram aplicadas entrevistas semiestruturadas a 20 integrantes-chave da Rota dos Butiazais. As entrevistas foram aplicadas de forma presencial a 9 pessoas (gravadas e transcritas pela pesquisadora) e via e-mail para 11 participantes (por motivo do distanciamento social, imposto pela pandemia do Covid-19). Foram utilizados: análise de conteúdo temática, de Laurence Bardin, e referencial teórico da Teoria das Representações Sociais de Serge Moscovici. Os participantes relataram sobre os compostos antioxidantes, fibras, carotenos, vitaminas e potássio presentes na polpa de butiá, expondo que estes compostos são benéficos para a saúde. Também trouxeram que o butiá tem valor nutritivo, é rico em vitamina $C$, e que podem existir propriedades que não tenham sido estudadas. Cada entrevistado tem uma história pessoal e familiar distinta, mas convergem em vários pontos relacionados ao conhecimento das propriedades do butiá benéficas à saúde. Os relatos dos participantes demonstram as representações sociais alicerçadas a partir do butiá.

\section{Palavras-Chave}

Teoria das Representações Sociais. Palmeira. Conhecimento popular.

\footnotetext{
${ }^{1}$ Doutora em Enfermagem pela Universidade Federal de Pelotas, Rio Grande do Sul, Brasil; fisioterapeuta. Email: portelinhamarcia@gmail.com.

2 Doutora em Genética e Biologia Molecular pela Universidade Federal do Rio Grande do Sul, Brasil; pesquisadora na Empresa Brasileira de Pesquisa Agropecuária, Pelotas, Rio Grande do Sul; professora colaboradora no Programa de Pós-Graduação em Agronomia, Universidade Federal de Pelotas, Rio Grande do Sul, Brasil. E-mail: lia.barbieri@embrapa.br.

${ }^{3}$ Doutora em Ciências pela Universidade Federal de Pelotas, Rio Grande do Sul, Brasil, com período sanduíche em Universitat Roivira i Virgili, Espanha; professora adjunta do Departamento de Enfermagem em Saúde Pública, Universidade Federal de Pelotas, Rio Grande do Sul, Brasil; membro do Núcleo de Pesquisa em Saúde Rural e Sustentabilidade. E-mail: teila.ceolin@gmail.com.
} 


\title{
The health-beneficial properties of butia from the perspective of key members of the Butia Palm Groves Network
}

\author{
Márcia Kaster Portelinha ${ }^{4}$, Rosa Lía Barbieri ${ }^{5}$, Teila Ceolin ${ }^{6}$
}

\begin{abstract}
The study aimed to describe the knowledge of the members of the Butia Palm Groves Network about the properties of butia that are beneficial to health. It was a qualitative research, with a descriptive approach, with data collection in March and April of 2020. Semistructured interviews were applied to 20 key members of the Rota dos Butiazais. The interviews were applied in person to nine people (recorded and transcribed by the researcher) and via e-mail to 11 participants (due to the social distance, imposed by the pandemic of Covid-19). Thematic content analysis by Laurence Bardin and the theoretical framework of the Theory of Social Representations by Serge Moscovici were used. Participants reported on the antioxidant compounds, fibers, carotenes, vitamins and potassium present in the butia pulp, stating that these compounds bring benefits for health. They also mentioned that butiá has a nutritional value, is rich in vitamin $\mathrm{C}$, and that there may be properties that have not been studied. Each interviewee has a distinct personal and family history, but they converge on several points related to the knowledge related to the properties of butiá. The participants' reports demonstrate the social representations based on the butiá.
\end{abstract}

\section{Keywords}

Theory of Social Representations. Palm tree. Popular knowledge.

\footnotetext{
${ }^{4} \mathrm{PhD}$ in Nursing, Federal University of Pelotas, State of Rio Grande do Sul, Brazil; physical therapist. E-mail: portelinhamarcia@gmail.com.

${ }^{5} \mathrm{PhD}$ in Genetics and Molecular Biology, Federal University of Rio Grande do Sul, State of Rio Grande do Sul, Brazil; researcher at the Brazilian Agricultural Research Corporation , Pelotas, State of Rio Grande do Sul; collaborating professor at the Postgraduate Program in Agronomy, Federal University of Pelotas, State of Rio Grande do Sul, Brazil. E-mail: lia.barbieri@embrapa.br.

${ }^{6} \mathrm{PhD}$ in Science, Federal University of Pelotas, State of Rio Grande do Sul, Brazil, with a sandwich period at Universitat Roivira i Virgili, Spain; adjunct professor at the Department of Public Health Nursing, Federal University of Pelotas, State of Rio Grande do Sul, Brazil; member of the Research Group on Rural Health and Sustainability. E-mail: teila.ceolin@gmail.com.
} 


\section{Introdução}

Butiazais são aglomerados de palmeiras que produzem cachos de frutos comestíveis, os butiás. Os butiás (Butia sp.) têm antigas ligações com a cultura e a história das pessoas que residem no Sul do Brasil. Essas palmeiras pertencem ao gênero Butia, que reúne 21 espécies na América do Sul, distribuídas no Brasil, Uruguai, Argentina e Paraguai (ESLABÃO et al., 2016). No Rio Grande do Sul, há oito espécies nativas de butiá, o que representa mais de um terço da diversidade total do gênero (WOLFF; WEGER; HEIDEN, 2016).

Na obra Da flora medicinal do Rio Grande do Sul, publicada no início do século 20 (D'ÁVILA, 1910), espécies de butiá já eram citadas como plantas medicinais de uso corrente, relatando-se benefícios no consumo.

$\mathrm{O}$ interesse pelo consumo de frutos nativos tem aumentado, pela valorização da biodiversidade local e pelos benefícios que seus compostos podem trazer para a saúde, o que tem impulsionado a busca por conhecimento a respeito das suas caracteristícas, tanto in natura quanto processados. Ao conhecer suas potencialidades e estimular o consumo das espécies nativas, esses recursos genéticos são valorizados e estimula-se sua preservação (FONSECA, 2012).

A humanidade vem construindo um conhecimento a respeito das plantas, utilizando-as de formas diversas, como no cuidado à saúde, no alimento, na moradia, no vestuário e também para o bem-estar. A partir de observações houve uma seleção cuidadosa na forma de uso das plantas, com tentativas e erros, sucessos e fracassos, que ao longo dos séculos, resultou no saber sobre as mesmas que é utilizado até hoje (HECK; RIBEIRO; BARBIERI, 2017).

O cultivo e o uso de plantas medicinais envolvem a educação popular em saúde, a qual promove encontros, vivências de gestão participativa, valorização das experiências, dos saberes e culturas, e práticas de cuidado populares (NESPOLI et al., 2021).

O Ministério da Saúde, em 2013, por meio da Portaria no 2.761, instituiu a Política Nacional de Educação Popular em Saúde no âmbito do Sistema Único de Saúde (PNEPSSUS), propondo uma prática político-pedagógica que perpassa as ações voltadas para a promoção, proteção e recuperação da saúde, a partir do diálogo entre a diversidade de saberes, valorizando os saberes populares, a ancestralidade, o incentivo à produção individual e coletiva de conhecimentos e a sua inserção no SUS (BRASIL, 2013).

Nesse contexto, a intenção de fortalecimento de redes voltadas à conservação da biodiversidade pelo uso sustentável, como a Rota dos Butiazais, vem contribuindo para o 
compartilhamento e o intercâmbio de experiências e de conhecimentos interdisciplinares, integrando-os às demandas relacionadas à saúde das comunidades (BRASIL, 2017).

A Rota dos Butiazais é uma rede internacional que se estruturou com base em um projeto de pesquisa liderado pela Empresa Brasileira de Pesquisa Agropecuária (Embrapa), buscando avanços no conhecimento e estabelecimento de conexões entre as pessoas que têm uma ligação com os butiás e os butiazais. É importante ressaltar que essas palmeiras representam uma alternativa econômica para extrativistas, agricultores, artesãos e pequenas agroindústrias que produzem e comercializam alimentos e artesanato a partir dos frutos, coquinhos e folhas (BARBIERI, 2015).

A Rota dos Butiazais aproxima-se da educação popular, almejando recuperar o valor da tradição, da experiência e da narrativa nos processos educativos, compreendendo que conhecer o universo cultural das pessoas, suas referências de mundo, seus vocabulários, seus ritos e costumes é fundamental para se estabelecer o diálogo e uma aprendizagem que faça sentido (NESPOLI et al., 2021).

Considerando que os butiazeiros são plantas nativas no Brasil, Uruguai, Argentina e Paraguai, e que apresentam potencial econômico e importância cultural no Bioma Pampa, este estudo teve o objetivo de descrever o conhecimento dos integrantes da Rota dos Butiazais a respeito das propriedades do butiá que são benéficas à saúde.

\section{Metodologia}

O trabalho foi desenvolvido de março a abril 2020, elaborado a partir do método qualitativo, com abordagem descritiva. O mesmo faz parte da tese de doutorado "Um olhar sobre a Rota dos Butiazais: inter-relações com a sustentabilidade e a saúde”.

Participaram da pesquisa 20 integrantes-chave da Rota dos Butiazais (parte das pessoas que idealizaram, construíram e mantém ativa a Rota dos Butiazais). Entre os selecionados foram incluídos artesãos, cozinheiros, ambientalistas, gestores públicos, pesquisadores, professores, empresários da área de turismo, proprietários rurais e extrativistas.

Foram realizadas entrevistas com 12 perguntas semiestruturadas. Conforme Santana e Lima (2020) apud Selltiz et al. (1987) esse tipo de entrevista permite um entrosamento entre o pesquisador e os entrevistados, e também possibilita a abordagem de um tema. A seleção dos participantes respeitou os seguintes critérios: ter idade superior a 18 anos, participar ativamente da Rota dos Butiazais, permitir gravar a entrevista e concordar com a divulgação e publicação dos resultados. Os critérios de exclusão foram: não aceitar responder a entrevista 
na sua totalidade, estar impossibilitado de se comunicar ou estar em atestado de saúde. O estudo foi organizado a partir da categoria: Propriedades do butiá e seus benefícios à saúde.

O método adotado foi Análise de Conteúdo, que é composta de um conjunto de instrumentos metodológicos cada vez mais sutis em constante aperfeiçoamento, que se aplicam a "discursos" (conteúdos) extremamente diversificados. Esta técnica oscila entre os dois polos, o do rigor da objetividade e o da fecundidade da subjetividade. Esta forma de análise pode classificar elementos em categorias. Com a investigação do que cada um deles tem em comum com outros, a parte comum existente entre eles resulta em seu agrupamento (BARDIN, 2016).

O trabalho foi balizado pelo referencial teórico de Moscovici (2015), que trata da Teoria das Representações Sociais. Essa teoria foi escolhida porque Serge Moscovici buscou compreender o sentido da produção de conhecimentos plurais, os quais constituem e reforçam as identidades dos grupos, influenciam nas suas práticas e reconstituem seus pensamentos (MOSCOVICI, 2015). O referencial teórico adotado auxiliou no entendimento a respeito da relação dos integrantes da Rota dos Butiazais com o butiá, com seu entorno e suas possibilidades.

Foram respeitados todos os preceitos éticos previstos na Resolução n ${ }^{\circ} 466 / 2012$, do Conselho Nacional de Saúde, do Ministério da Saúde, que dispõe sobre Pesquisas com seres humanos (BRASIL, 2012). A proposta do trabalho foi aprovada pelo Comitê de Ética em Pesquisa da Santa Casa de Misericórdia Pelotas sob o parecer número 3.926.894.

\section{Resultados e Discussão}

Os dados deste estudo abordam o conhecimento popular dos 20 participantes a respeito das propriedades do butiá que são benéficas à saúde, fatores nutricionais e seu potencial na melhoria de qualidade de saúde e consequentemente de vida. Nesse contexto, as ações da Rota dos Butiazais vêm incentivando outros grupos no sentido de conectar distintos atores que têm em comum o vínculo cultural com elementos da biodiversidade, através do fortalecimento das suas possibilidades e riquezas (PORTELINHA et al. 2020).

Sendo assim, pode-se dizer que a Rota do Butiazais também permeia os princípios da Política Nacional de Educação Popular em Saúde de diálogo, amorosidade, problematização, construção compartilhada do conhecimento, emancipação e compromisso com a construção do projeto democrático e popular (BRASIL, 2013). 
As pessoas não são receptores passivos, ao contrário, são sujeitos que procuram refazer, reorganizar a realidade que se apresenta diante deles por meio de um processo de transformação do novo ou diferente em algo conhecido e compatível com seu mundo de compreensão, essa organização é denominada, por Moscovici (2012), de ancoragem. Nesta ideia, os entrevistados deste estudo estão repensando, repassando e reavaliando as formas e contextos com que utilizam o butiá, no intuito de mudar e agregar novos conhecimentos às suas realidades e com isso ampliar as possibilidades de utilização e manuseio para com a planta, possibilitando a modificação das práticas, trazendo consigo novos rumos com perspectivas emancipatórias no sentido de agregação de renda.

As representações sociais trazem como segundo mecanismo o objetivo de transformar algo abstrato em algo quase concreto, transferir o que está na mente para algo que exista no mundo físico (MOSCOVICI, 2015). Nos depoimentos dos integrantes-chave da Rota dos Butiazais, os saberes estavam no campo abstrato, alguns conhecimentos eram familiares, estavam armazenados nas lembranças, realidade que foi sendo remanejada e trazida para o dia-a-dia, foi sendo customizada, e se transformando em algo real, com a possibilidade de trabalho, de melhoria de condições financeiras, e com isso proporcionando cada vez mais novos olhares, novas pactuações, aprendizados, troca e soma de saberes, e com isso o manuseio do butiá está se tornando cada vez mais conhecido e apreciado.

Nesse pensamento, o conhecimento é sempre produzido por meio da interação e comunicação, e sua expressão está sempre ligada aos interesses humanos que estão nele implicados (MOSCOVICI, 2015). Ideia que retrata a realidade abordada nos relatos dos integrantes deste estudo, o trabalho realizado com o butiá carrega o interesse em relação à planta, tanto relacionado à preservação cultural, quanto à parte histórica, então a soma das intenções potencializa a preservação do butiá e do seu entorno, pois ele passa do patamar de planta do quintal, para, com a diversificação dos produtos, trazer uma perspectiva de melhoria na renda familiar.

A educação popular valoriza os modos de cuidar oriundos da experiência, inclusive os que fazem uso de plantas, como práticas populares de cuidado (NESPOLI et al., 2021). As plantas medicinais foram contempladas, entre outras práticas integrativas e complementares, a partir de 2006, pela Política Nacional de Práticas Integrativas e Complementares (PNPIC) (BRASIL, 2015).

O olhar dos integrantes-chave da Rota dos Butiazais se dá no contexto da vida diária, entendendo que suas vivências, suas escolhas e utilização das riquezas do meio ambiente vão 
refletir diretamente na saúde das populações. Os participantes referiram diversos benefícios à saúde a partir do butiá, os quais serão abordados a seguir.

Os integrantes-chave relataram acerca das "Propriedades do butiá benéficas à saúde", que proporcionam "equilíbrio da saúde": compostos antioxidantes, fibras, carotenos, vitaminas e potássio, que previne cãibras, expondo que estas propriedades do butiá são benéficas para a saúde, inclusive para deixar a pessoa equilibrada. Também trouxeram que o butiá tem grande valor nutritivo, é rico em vitamina $\mathrm{C}$.

Existem compostos antioxidantes, fibras e vitaminas que são benéficas à nossa saúde. (CNP-pesquisador).

Também é antioxidante, a princípio tem potássio, pode prevenir câimbras. (RCV-artesão).

Olha, rico em vitamina C... Acho que ao comer ele na sua época certa, além de alimentar, ele vai estar te deixando bem equilibrado, eu costumo assim, que até é um questionamento que a gente vem fazendo também, o que é o medicinal? Porque para mim o remédio, é aquilo que tu comes. (MMGambientalista).

Tem propriedades sim, que eu acho que a gente ainda não sabe, talvez não tenham sido estudadas. (CHB-agropecuarista).

Os informantes relataram várias indicações do butiá, fruta que faz parte da natureza e é estudada nas suas características alimentares, benefícios para a saúde, podendo também proporcionar o equilíbrio; existem pesquisas em andamento buscando a exploração de mais propriedades. Em contrapartida, houve participantes que comentaram não saber sobre as propriedades da planta.

Várias publicações relatam que a polpa do butiá é rica em vitamina $\mathrm{C}$ e carotenoides, que são substâncias com atividade antioxidante, ou seja, atuam na manutenção da saúde (FRANCO 1999; MANELA-AZULAY et al., 2003; ZHANG; HAMAUZU, 2004; ROSA et al., 2007; FARIA et al., 2008; SGANZELA, 2010; FONSECA, 2012; RIVAS, M.; BARBIERI, R. L., 2014; CRUZA et al., 2017; MARTINS et al., 2019). Os butiás também apresentam altas concentrações de potássio, o qual é importante para regular o funcionamento do organismo (FARIA et al., 2008; RIVAS, M.; BARBIERI, R. L., 2014). Sua polpa apresenta altos níveis de minerais, como, por exemplo, ferro e manganês (SGANZELA, 2010; RIVAS; BARBIERI, 2014; PADILHA et al., 2016).

A partir das entrevistas descritas, e da discussão realizada com autores que abordam as características do butiá, verifica-se a proximidade dos saberes, mostrando que o conhecimento 
popular dos integrantes da Rota dos Butiazais vem acompanhando os estudos realizados, assimilado esse conhecimento e integrando-o nas falas.

A partir dos relatos dos participantes deste estudo, percebe-se que o estímulo ao uso de espécies nativas como alimento e fonte de vitaminas seria uma alternativa para reduzir a ameaça de extinção, promover a manutenção e o replantio dos butiazeiros (NUNES et al., 2008).

O butiá é um recurso genético que vem sendo utilizado há muito tempo, conforme mostram os registros arqueológicos e relatos da literatura. Porém, como muitas frutas nativas brasileiras, tem muito ainda a ser pesquisado a respeito pela comunidade científica (SCHWARTZ, 2008).

$\mathrm{O}$ aspecto nutricional do butiá é expressivo. O consumo dos frutos ocorre in natura, na forma de polpa congelada e de produtos obtidos por processamento, como bebidas e geleias. A polpa de butiá é uma opção de alimento saudável, pois além de ser um produto da biodiversidade local, é cheio de sentidos culturais e históricos (MARTINS et al., 2019). Os butiás são nutritivos e saborosos, mostrando grande potencial, inclusive de geração de renda (RIVAS; BARBIERI, 2014; PADILHA et al., 2016).

De acordo com as necessidades diárias de vitaminas recomendadas pelo Instituto Americano de Medicina (IOM, 2001) um copo de suco contendo 100g de polpa de butiá poderia suprir cerca de $40 \%$ das necessidades diárias de vitamina A (300-400 RAE dia-1) para crianças menores de 8 anos de idade e $100 \%$ das necessidades diárias de vitamina $\mathrm{C}$ (45mg dia-1) para homens e mulheres (FARIA, et al., 2008).

Os resultados obtidos demonstram o elevado potencial da polpa do butiá para enriquecer a alimentação da população, especialmente como fonte de fibras e vitaminas, a exemplo do que já vem sendo feito na merenda escolar no norte de Minas Gerais. Esses resultados também respaldam a importância cultural da espécie e da manutenção da variabilidade no Cerrado (FARIA et al., 2008; SGANZERLA, 2010). A colheita do butiá permite a produção da polpa congelada, que também é destinada à merenda escolar, favorece a geração de renda, enriquece a alimentação das comunidades locais e estimula a preservação da espécie (FARIA et al., 2008).

Os integrantes-chave também fizeram referência às "propriedades da amêndoa do butiá”, sobre o óleo extraído da amêndoa, seu valor nutritivo e os benefícios para a pele.

E a amêndoa, além de ser muito saborosa, ela tem um valor nutritivo muito grande, então tem propriedades sim. (CHB-agropecuarista). 
Tem um óleo muito bom extraído da amêndoa, maravilhoso, bom para pele. (MAB- empresária).

Fiquei sabendo do chazinho, agora nessa semana fiquei sabendo que estão fazendo chazinho da amêndoa. (FVA-extensionista rural).

As sementes do butiá contêm óleo de alta qualidade, que pode ser utilizado no desenvolvimento de novos produtos em diferentes setores da indústria, como alimentícia, farmacêutica ou cosmética (RIVAS; BARBIERI, 2014). As amêndoas têm efeito antihelmíntico (RODRIGUES et al., 2018), são ricas em óleos, e são consumidas na forma de biscoitos, tortas, bombons e de uma infusão conhecida como "café de coco" (TONIETTO; SCHLINDWEIN; TONIETTO, 2009).

As sementes de butiá são matéria-prima interessante para fornecer extratos com substâncias biologicamente ativas, como agentes antibacterianos mais eficazes contra bactérias Gram-negativas em comparação com as Gram-positivas (CRUZA et al., 2017). O óleo essencial extraído das sementes de butiá apresentou efeito antimicrobiano, com potencial ação contra bactérias orais. Nesse contexto, a incorporação de óleos essenciais pode ser uma alternativa ecológica, sustentável e um material de baixo custo (PERALTA, 2011; PERALTA et al., 2013; SUN et al., 1988; SZENTMIHALYI et al., 2002). O óleo essencial desse fruto chama atenção por apresentar um potencial agente antimicrobiano contra bactérias orais (SUN et al., 1988; PERALTA et al., 2013). Além disso, outra vantagem do uso da semente do butiá como matéria-prima para obtenção de óleos essenciais com características antimicrobianas seria que ela é uma fonte renovável e um material de baixo custo (SZENTMIHALYI et al., 2002).

Um resgate cultural de conhecimento popular sobre plantas no município de Mafra (SC), realizado através de entrevistas, possibilitou verificar que a maioria das plantas utilizadas por moradores apresenta embasamento científico em relação à indicação de seu uso. Neste sentido, se acredita na relevância de estudos como este na área de saúde pública, pois permitem aproximar a academia da população (HUMENHUK et al., 2020). Assim, estudos como este trazem que abordam o conhecimento popular, influenciando na qualidade da saúde e nas práticas cotidianas.

Os integrantes-chave também se referiram "à terapia floral com base na essência do butiá", a qual auxilia a despertar forças de defesa, trabalha o primeiro e o terceiro chakras, ajudando no empoderamento, com ação sobre o fígado, além de lidar com a raiva. 
Pela terapia floral, a essência da planta butiá, [...], ela ajuda a despertar as forças, de defesa. É uma essência que trabalha muito o primeiro e o terceiro chakras, ajuda no empoderamento, proporciona limpezas necessárias no fígado, [...]. É uma essência que ajuda a lidar com a raiva, que pode ser um veneno, mas que também é necessária. A raiva é um sentimento bom, ela é um sentimento positivo, quando bem administrado. (CHB-agropecuarista).

O que eu vi muito foi nas pesquisas dos florais. Utiliza essências florais que vêm do butiazal, e com certeza o floral é um equilíbrio para a saúde. (JVambientalista).

As Essências do Butiazal têm por objetivo auxiliar na busca de equilíbrio e desenvolvimento das pessoas. A terapia de florais é uma prática complementar e não medicamentosa que, por meio das essências florais, modifica estados vibratórios buscando equilibrar e harmonizar o indivíduo. A essência floral é extraída da planta em floração, atua estimulando transformação positiva na forma do pensamento $\mathrm{e}$ propiciando $\mathrm{o}$ desenvolvimento interior, equilíbrio emocional que conduz a novos comportamentos. Neste contexto, a Organização Mundial da Saúde (OMS) preconiza o reconhecimento e incorporação das Práticas Integrativas e Complementares nos sistemas nacionais de saúde (BRASIL, 2018).

As Essências do Butiazal são inspiradas em um butiazal localizado no município de Tapes/RS e que tem sido cenário de pesquisas científicas promovidas sobre conservação e uso sustentável do ecossistema pela Embrapa Clima Temperado (GAV, 2011).

Neste contexto, averiguou-se que o interesse dos integrantes-chave da Rota dos Butiazais resgata, mantém e gera conhecimento a respeito do butiá, demonstrando saberes entrelaçados com a planta.

Estudos como esse permitem que o pesquisador identifique saberes, representações sociais a respeito dos mais diversos aspectos. Nesse caso, as propriedades do butiá que são benéficas à saúde, assunto que impulsiona essa rede de pessoas e traz sentido às aproximações, pactuações e relações que balizam a Rota dos Butiazais.

\section{Considerações finais}

No resgate do conhecimento popular sobre as propriedades do butiá benéficas à saúde a maioria dos participantes trouxe saberes anteriores, familiares, que quando aproximados de bibliografias sobre o tema, demonstram um alinhamento de saberes, os quais deflagram a soma e acúmulo de saberes que perpassa tanto o saber popular, quanto o conhecimento científico. 
A Rota dos Butiazais demonstra as representações sociais alicerçadas a partir do butiá, e que convergem em vários pontos, mostrando saberes, intenções de preservação cultural e história, que abarcam também o entorno da planta.

A subjetividade dos dados, os saberes populares, a ancestralidade e a carga afetiva, impulsiona o diálogo entre os diversos saberes, e todo esse contexto faz com que a descrição desse formato de estudo seja complexa, pois muitas dessas informações, resgatadas do conhecimento popular, estavam nos pensamentos, e quando começam a ser organizadas, estruturadas e disponibilizadas, vão consequentemente sendo passadas para o saber científico.

\section{Agradecimentos}

Agradecemos o apoio financeiro do Ministério da Ciência, Tecnologia e Inovações (MCTI) e do Conselho Nacional de Desenvolvimento Científico e Tecnológico (CNPq) ao projeto da Rota dos Butiazais (processo 441493/2017-3).

\section{Referências}

BARDIN, L. Análise de conteúdo. São Paulo: Edições 70, 2016.

BARBIERI, R. L. et al. Vida no butiazal. Brasília: Embrapa, 2015.

BRASIL. Ministério da Saúde. Portaria no 2.761, de 19 de novembro de 2013. Institui a Política Nacional de Educação Popular em Saúde no âmbito do Sistema Único de Saúde (PNEPS-SUS). Brasília, DF: Ministério da Saúde, 2013. Disponível em: https://bvsms.saude.gov.br/bvs/saudelegis/gm/2013/prt2761_19_11_2013.html Acesso em: 20 out. 2021.

BRASIL. Ministério da Saúde. Resolução CNS no 466, de 12 dezembro de 2012. Brasília, DF: Conselho Nacional de Saúde. 2012. Disponível em: https://bvsms.saude.gov.br/bvs/saudelegis/cns/2013/res0466_12_12_2012.html. Acesso em: 12 jan. 2021.

BRASIL. Ministério da Saúde. Secretaria de Ciência, Tecnologia e Insumos Estratégicos. Departamento de Assistência Farmacêutica. Política nacional de plantas medicinais e fitoterápicos Brasília. DF: Ministério da Saúde, 2006. Disponível em: http://bvsms.saude.gov.br/bvs/publicacoes/politica_nacional_fitoterapicos.pdf. Acesso em: 10 dez. 2020.

BRASIL. Ministério da Saúde. Secretaria de Atenção à Saúde. Departamento de Atenção Básica. Política nacional de práticas integrativas e complementares no SUS: atitude de ampliação de acesso 2. ed. Brasília, DF: Ministério da Saúde, 2015. Disponível em: 
http://bvsms.saude.gov.br/bvs/publicacoes/politica_nacional_praticas_integrativas_compleme ntares_2ed.pdf. Acesso em: 20 jan. 2021.

BRASIL. Diretoria de Ciências Agrárias, Biológicas e da Saúde. Conselho Nacional de Desenvolvimento Científico e Tecnológico DABS. Nexus II: Pesquisa e Desenvolvimento em Ações Integradas e Sustentáveis para a Garantia da Segurança Hídrica, Energética e Alimentar nos Biomas Pampa, Pantanal e Mata Atlântica. Embrapa Clima Temperado. Pelotas, RS, 2017. Disponível em: https://www.ufsm.br/grupos-de-pesquisa/nexuspampa/wpcontent/uploads/sites/364/2018/04/Projeto-Nexus-Pampa-II.pdf. Acesso em: 11 mar. 2021.

BRASIL. Ministério da Saúde. Portaria no 702, de 21 de março de 2018. Disponível em: https://www.in.gov.br/web/guest/materia/-

/asset_publisher/Kujrw0TZC2Mb/content/id/7526450/do1-2018-03-22-portaria-n-702-de-21de-marco-de-2018-7526446.

Acesso em: 10 fev. 2021.

CRUZA, P. N. et al. Antioxidant and antibacterial potential of butia (Butia catarinensis) seed extracts obtained by supercritical fluid extraction. The Journal of Supercritical Fluids, [s. l.] v. 119, p. 229-237, 2017. Doi: 10.1016/j.supflu.2016.09.022. Disponível em: https://www.sciencedirect.com/science/article/abs/pii/S0896844616303436. Acesso em: 15 mar. 2021.

D’ÁVILA, M. C. Da flora medicinal do Rio Grande do Sul. Porto Alegre: Faculdade de Medicina e Pharmácia de Porto Alegre, 1910.

ESLABÃO, M. P. et al. Mapeamento da distribuição geográfica de butiá como subsídio para a conservação de recursos genéticos. Pelotas: Embrapa, 2016. (Boletim de Pesquisa e Desenvolvimento, n. 252).

FARIA, J. P. et al. Caracterização da polpa do coquinho-azedo (Butia capitata var capitata).

Rev. Bras. Frutic., Jaboticabal, v. 30, n. 3, p. 827-829, 2008. Doi: 10.1590/S010029452008000300045. Disponível em: https://www.scielo.br/scielo.php?pid=S010029452008000300045\&script=sci_abstract\&tlng=pt. Acesso em: 22 dez. 2020.

FONSECA, L. X. Caracterização de frutos de butiazeiro (Butia odorata Barb. Rodr.) Noblick \& Lorenzi e estabilidade de seus compostos bioativos na elaboração e armazenamento de geleias. 2012. 68 f. Dissertação (Mestrado em Ciência e Tecnologia Agroindustrial) - Faculdade de Agronomia Eliseu Maciel, Universidade Federal de Pelotas, Pelotas, 2012. Disponível em: http://guaiaca.ufpel.edu.br:8080/handle/prefix/5923. Acesso em: 15 maio 2021.

FRANCO, G. Tabela de composição química de alimentos. 10. ed. Rio de Janeiro: Atheneu. 1999.

GAV. Grupo de Aprimoramento da Vida. 2011. Disponível em: https://gav.org.br/missaovisaoevalores/. Acesso em: 25 mar. 2021.

HECK, R. M.; RIBEIRO, M. V.; BARBIERI, R. L. Plantas medicinais do Bioma Pampa no cuidado em saúde. Brasília, DF: Embrapa, 2017. 
HUMENHUK T.; LEITE D. R. B.; FRITSCH M. Conhecimento popular sobre plantas medicinais utilizadas no município de Mafra, SC, Brasil. Saúde Meio Ambient., Constestado, v. 9, p. 27-42, 2020. Doi: 10.24302/sma.v9i0.2466. Disponível em: https://www.periodicos.unc.br/index.php/sma/article/view/2466. Acesso em: 20 jan. 2021.

MANELA-AZULAY, M. et al. Vitamina C. Anais Dermatologia Brasileira, Rio de Janeiro, v. 78, n. 3, p. 265-274, 2003. Doi: 10.1590/S0365-05962003000300002. Disponível em: http://www.anaisdedermatologia.org.br/. Acesso em: 10 mar. 2021.

MARTINS, J. S. et al. Avaliação nutricional de Butiá (Butia yatai) processado. Segurança Alimentar e Nutricional, Campinas, v. 26, p. 1-7, 2019. Doi: 10.20396/san.v26i0.8654389. Disponível em: https://periodicos.sbu.unicamp.br/ojs/index.php/san/article/view/8654389. Acesso em: 20 nov. 2020.

MOSCOVICI, S. Representações sociais: investigações em Psicologia Social. 11. ed. Petrópolis-RJ: Vozes, 2015.

MOSCOVICI, S. A psicanálise, sua imagem e seu público. Petrópolis-RJ: Vozes, 2012.

NESPOLI, G. et al. (org.). Educação popular e plantas medicinais na atenção básica à saúde. Rio de Janeiro: EPSJV, 2021.

NUNES, A. M. et al. Caracterização molecular de butiazeiro por marcadores. Revista Brasileira de Fruticultura, Jaboticabal, v. 30, n. 3, p. 702-707, 2008. Doi: 10.1590/S010029452008000300024. Disponível em:

https://www.scielo.br/j/rbf/a/nxLdrSY4SSt8cgwXXWyPpNP/?lang=pt. Acesso em: 15 mar. 2021.

PADILHA, H. K. M. et al. Avaliação da produção de cachos de frutas em palmeiras de Butiá (Butia odorata (Barb. Rodr.) Noblick \& Lorenzi). Magistra, Cruz das Almas, v. 28, p. 419426, 2016. Disponível em:

https://www.researchgate.net/publication/320962520_Avaliacao_da_producao_de_cachos_de _frutas_em_palmeiras_de_Butia_Butia_odorata_Barb_Rodr_Noblick_Lorenzi. Acesso em: 13 jan. 2021.

PERALTA, S. L. Incorporação do óleo essencial de Butia capitata como antibacteriano em um adesivo experimental. 2011. 116 f. Dissertação (Mestrado em Odontologia) Universidade Federal de Pelotas, Pelotas, 2011. Disponível em:

http://guaiaca.ufpel.edu.br:8080/handle/123456789/2230. Acesso em: 10 maio 2021.

PERALTA, S. L. et al. Self-etching dental adhesive containing a natural essential oil: antibiofouling performance and mechanical properties. Biofouling, [s. l.], v. 29, n. 4, p. 345-55, 2013. Doi: 10.1080/08927014.2013.770477. Disponível em:

https://pubmed.ncbi.nlm.nih.gov/23560741/. Acesso em: 10 maio 2021.

PORTELINHA, M. K. et al. Os butiazeiros e a Rota dos Butiazais, sementes de cuidado e conexão. Vittalle, Carreiros, v. 32, n. 1, p. 135-145, 2020. Doi:

10.14295/vittalle.v32i1.11257. Disponível em:

https://ainfo.cnptia.embrapa.br/digital/bitstream/item/218301/1/11257-Texto-do-artigo35612-1-10-20200720.pdf. Acesso em: 24 mar. 2021. 
RIVAS, M.; BARBIERI, R. L. Boas práticas de manejo para o extrativismo sustentável do Butiá. Pelotas: Embrapa Clima Temperado, 2014.

RODRIGUES, E. E. et al. Plantas del bajo Río Uruguay Árboles y Arbustos. Comisión Administradora del Río Uruguay, v. 1, Paysandú, 2018. Disponível em:

https://www.researchgate.net/publication/329502908_Plantas_del_bajo_Rio_Uruguay_ARBO LES_y_ARBUSTOS. Acesso em: 10 mar. 2021.

ROSA, J. S. et al. Desenvolvimento de um método de análise de vitamina C em alimentos por cromatografia líquida de alta eficiência e exclusão iônica. Food Science and Technology, Campinas, v. 27, n. 4, p. 837-846, dez. 2007. Doi: 10.1590/S0101-20612007000400025 . Disponível em: https://www.scielo.br/j/cta/a/z9gS48S4L8m YhFmnmGbvqbL/?lang=pt. Acesso em: $1^{\circ}$ maio 2021.

SANTANA, E. L. C.; LIMA, T. P. P. Contribuições da educação ambiental para a cidadania. Revista de Educação Popular, Uberlândia, v. 19, n. 3, p. 158-170, 2020. Doi: 10.14393/REP-2020-52901. Disponível em: http://www.seer.ufu.br/index.php/reveducpop/article/view/52901/30294. Acesso em: 5 jan. 2021.

SCHWARTZ, E. Produção, fenologia e qualidade dos frutos de Butia capitata em populações de Santa Vitória do Palmar. 2008. 92 f. Tese (Doutorado em Fruticultura de Clima Temperado) - Universidade Federal de Pelotas, Pelotas, 2008. Disponível em: http://repositorio.ufpel.edu.br:8080/bitstream/123456789/2117/1/Tese_\%20Elisane_\%20Sch wartz.pdf. Acesso em: 10 abr. 2021.

SELLTIZ, C. et al. Métodos de pesquisa nas relações sociais. Tradução de Maria Martha Hubner de Oliveira. 2. ed. São Paulo: EPU, 1987.

SGANZERLA, M. Caracterização físico-química e capacidade antioxidante do butiá. 2010. 105 f. Dissertação (Mestrado em Ciência e Tecnologia Agroindustrial) - Universidade Federal de Pelotas, Pelotas, 2010. Disponível em:

https://dctaufpel.com.br/ppgcta/manager/uploads/thesis/mest_marla_sganzerla.pdf. Acesso em: 10 abr. 2021.

SUN, C. et al. Acyl coenzyme a preference of the glycerol phosphate pathway in the microsomes from the maturing seeds of palm, maize, and rapeseed. Plant Physiology, Oxford, n. 1, p. 56-60, 1988. Doi: 10.1104/pp.88.1.56. Disponível em: https://pubmed.ncbi.nlm.nih.gov/16666279/. Acesso em: 10 mar. 2021.

SZENTMIHALYI, K. et al. Rose hip (Rosa canina L.) oil obtained from waste hip seeds by different extraction methods. Bioresour Technol, [s. l.], v. 82, n. 2, p. 195-201, 2002. Doi: 10.1016/s0960-8524(01)00161-4. Disponível em:

https://www.sciencedirect.com/science/article/abs/pii/S0960852401001614. Acesso em: 20 mar. 2021.

TONIETTO, A.; SCHLINDWEIN, G.; TONIETTO, S. M. Usos e potencialidades do butiazeiro. Porto Alegre: Fepagro, 2009. (Circular Técnica. n. 26). disponível em: https://issuu.com/fepagro/docs/circular_26. Acesso em: 17 nov. 2020. 
WOLFF, L. F.; WEGNER, J.; HEIDEN, G. Butiazeiros como flora apícola para a produção de mel na região sul do Rio Grande do Sul. Pelotas: Embrapa, 2016. (Boletim de Pesquisa e Desenvolvimento. n. 25342). Disponível em:

http://www.bdpa.cnptia.embrapa.br/consulta/busca?b=ad\&id=1081697\&biblioteca=vazio\&bu $\mathrm{sca}=1081697 \& q$ Facets $=1081697 \&$ sort $=\&$ paginacao $=t \&$ paginaAtual $=1$. Acesso em: 05 dez . 2020 .

ZHANG, D.; HAMAUZU, Y. Phenolics, ascorbic acid, carotenoids and antioxidant activity of broccoli and their changes during conventional and microwave cooking. Food Chemistry, s. l.], v. 88, n. 4, p. 503-509, 2004. Doi: 10.1016/j.foodchem.2004.01.065. Disponível em: https://www.sciencedirect.com/science/article/abs/pii/S0308814604001517. Acesso em: 14 de fev. 2021.

Submetido em 4 de junho de 2021.

Aprovado em 8 de novembro de 2021. 